\title{
A cystic cerebral tuberculoma treated surgically
}

\author{
H. M. DASTUR, A. D. DESAI, AND DARAB K. DASTUR ${ }^{1}$ \\ From the Department of Neurosurgery, K.E.M. Hospital, and the Neuropathology \\ Unit of the I.C.M.R., Tata Memorial Hospital, Bombay, India
}

In the lesser developed countries of Africa and Asia tuberculosis is widely prevalent in all its various forms and still presents a major health problem. It is therefore not surprising that 20 to $25 \%$ of brain tumours prove to be tuberculomas so that this condition is not rare as it is in the West. In this department, which has been functioning now for over five years, more than 95 patients have already undergone operative excision of brain tuberculomas. In a histopathological analysis of 452 intracranial spaceoccupying lesions studied over the past eight and a half years at the Neuropathology Unit (Dastur and Iyer, 1961), 102 or $23 \%$ were found to be tuberculomas. The great majority of these have conformed to the types described in standard works on neurology and neuropathology (Cushing, 1932; Courville, 1945; Dublin, 1954; Kinnier Wilson, 1954; Greenfield, Blackwood, McMenemey, Meyer, and Norman, 1958). Our reason for publishing this solitary case is that it does not conform to any of these descriptions or even to any in large operative series such as those of Arseni (1958) and Ramamurthi and Varadarajan (1961).

Instead of the usual multifocal, caseous or noncaseous type of mass, the major portion of this tumour consisted of a cystic change, a state of affairs which we have never before seen, nor indeed thought possible. During the operation it was quite apparent that this was not a cystic glioma, and having on one previous occasion seen a similar cystic change in a temporal lobe chordoma (Dastur, 1961), we not unnaturally suspected a chordoma rather than a tuberculoma. However, microscopic examination of the tumour left no doubt as to its identity.

\section{CLINICAL FEATURES}

A.B., a boy aged 4 years, was first admitted to a general surgical unit at this hospital on 5 July 1959, with a history of having had a fall on the same day while playing at home. He was said to have become unconscious and developed a right hemiparesis following what the parents described as convulsive movements. By the morning after admission the child had regained consciousness and was able to take

${ }^{1}$ Present address: Postgraduate Research Laboratories, J. J. Group of Hospitals, Bombay 8. feeds, but the right hemiparesis persisted. Skull radiographs were normal and after a fortnight's stay in the ward he was discharged and referred to the Neurological Unit for out-patient treatment. An E.E.G. was performed and as this indicated an atrophic lesion, a pneumoencephalogram was advised but the parents refused to have the child admitted, although they continued to bring him to the out-patient clinic regularly for anticonvulsant treatment till November 1960, after which treatment was discontinued altogether.

The patient was seen again on 14 April 1961, having had for the previous 15 days attacks of severe headache, vomiting, and generalized convulsions. Ten days after this visit, the parents agreed to have the child admitted to the Neurosurgical Unit.

On examination he was drowsy, unresponsive, and incontinent. The skull was considerably enlarged with a circumference of $22 \frac{1}{2} \mathrm{in}$. and the sutures were widely separated so that he could barely support the weight of his head. Examination of the fundus revealed bilateral papilloedema associated with secondary optic atrophy and vision appeared to be grossly impaired. In addition he had a severe right hemiparesis. An E.E.G. on this occasion showed further deterioration, but in the presence of a rapidly worsening clinical picture it was felt that the changes observed indicated the presence of a massive left frontal tumour rather than advancing atrophy.

On the day following admission his condition deteriorated suddenly, with loss of consciousness, a high temperature with rapid pulse and respiratory rates (temperature $104 \cdot 6^{\circ} \mathrm{F}$., pulse 150 , respiration 40 ).

A right posterior parietal burr hole was made and a grossly distended lateral ventricle drained through an indwelling rubber catheter. This produced an immediate improvement in the level of consciousness and the drain was maintained over the next three days, the average daily collection of cerebrospinal fluid amounting to $150 \mathrm{ml}$.

A ventriculogram (Fig. 1) was recorded on the fifth day. Through the catheter air was introduced into the right lateral ventricle which appeared enormously dilated, and although some air passed into the third ventricle, it could not be manipulated across to the left, presumably due to obstruction of the left foramen of Monro. To demonstrate the left ventricle, a posterior parietal burr hole was made on the left side and a brain needle advanced in the direction of the lateral ventricle only to encounter at a depth of $3 \mathrm{~cm}$. a firm capsule which was punctured and immediately there was a spurt of oily yellow transparent fluid which began to clot rapidly on 


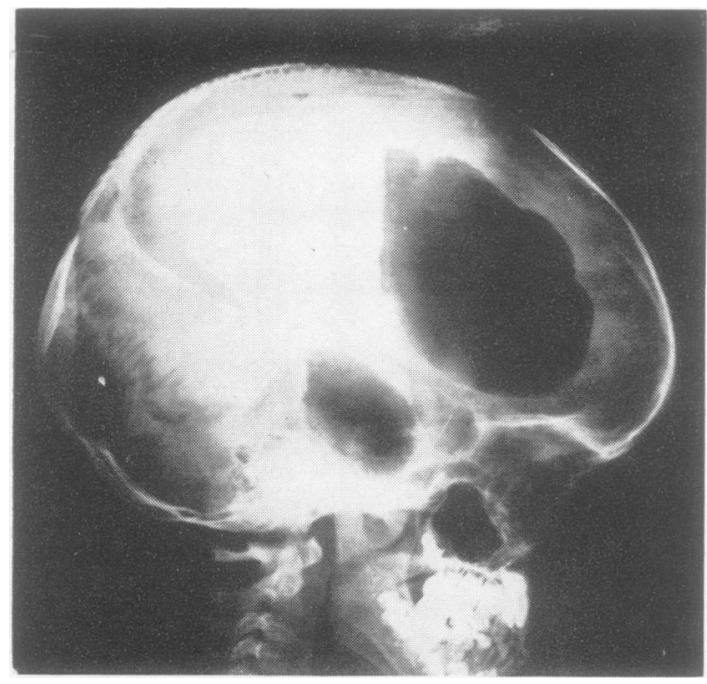

FIG. 1. Brow-up lateral ventriculogram showing air in the right frontal and temporal horns and outlining faintly the anterior part of the third ventricle.

standing. About $100 \mathrm{ml}$. of fluid was thus evacuated and replaced by about $60 \mathrm{ml}$. of air to obtain a cystogram. This (Fig. 2) showed a large globular parietal shadow with a filling defect in its floor, not in communication with the left lateral ventricle which did not fill. A provisional diagnosis of cystic glioma was made but as the patient was again desperately ill with a rise of temperature to $104^{\circ} \mathrm{F}$., pulse to $180 / \mathrm{min}$., and respiration to $50 / \mathrm{min}$., craniotomy was postponed.

On the seventh day he rallied and after the temperature was reduced to normal by combined icing and a lytic cocktail, he was taken to the theatre for craniotomy.

CRANIOTOMY Through a left fronto-parietal flap extending forwards to the coronal suture, the dura was exposed and the cyst tapped, reducing considerably the intra- cranial tension. A curved dural flap was cut and reflected upwards. The exposed convolutions were uniformly expanded, but in addition, those in the anterior half were bluish-grey and translucent, contrasting vividly with the normal colouring of the convolutions further back. At this stage it was difficult to decide whether this was oedema, as is sometimes seen around a tuberculoma, or invasion by glioma. A cortical incision was made and the antero-lateral wall of the cyst seen at a depth of $1 \mathrm{~cm}$. By blunt dissection a large part of the cyst wall was exposed, and in doing so multiple small peripheral loculated arachnoid cysts were opened. The cyst wall was 3 to $4 \mathrm{~mm}$. thick and encrusted with yellow patches of calcification. On cutting into the cyst there was a large cavity extending backwards, while in the front there was a craggy, irregular, dense white mass curving backwards along the floor of the cyst. It was almost entirely avascular and was excised with a pair of stout scissors. The cyst wall was now grasped with rongeurs and by traction and gentle dissection all of it was excised. The tumour weighed $50 \mathrm{~g}$. and in its gross appearance bore a strong resemblance to a cystic chordoma, which we had once previously had occasion to excise (Dastur, 1961). Parts of the frontal horn and body of the lateral ventricle, which were opened during the excision, were seen communicating with the tumour bed.

POST-OPERATIVE COURSE From the second post-operative day the child began to talk and take feeds by mouth and since then improvement has continued uninterruptedly. He was discharged three months after the operation and throughout this period he received streptomycin $1 \mathrm{~g}$., isoniazid $200 \mathrm{mg}$., and P.A.S. 8 g. per day. When he left the hospital he was alert, bright, playful, putting on weight, and had normal speech. Fundi showed secondary optic atrophy but vision was adequate, as he could count fingers and identify small objects, including coins, at three metres. He could stand without support and was learning to walk with assistance. The upper limb had largely recovered but there was still incoordination and clumsiness of finger movements.

At routine screening of the members of his family, his father was found to be suffering from pulmonary tuber-

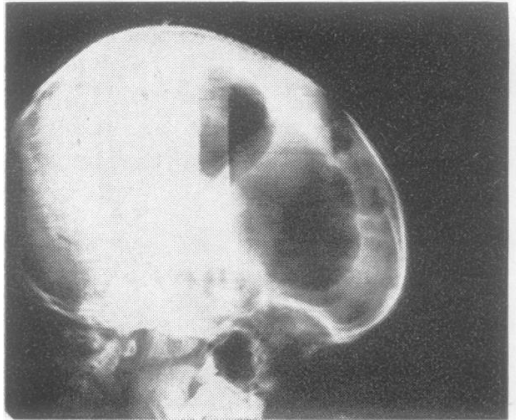

A

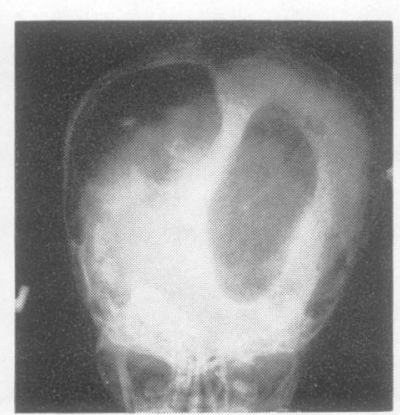

B

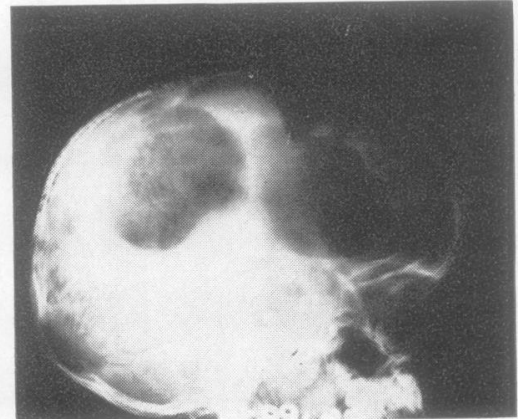

$U^{\prime}$

FIG. 2. Ventriculogram and cystograms showing right lateral ventricle and left parietal cyst in three views. A, brow-up lateral. B, half axial. C, lateral with left side uppermost, outlining the entire cyst. 


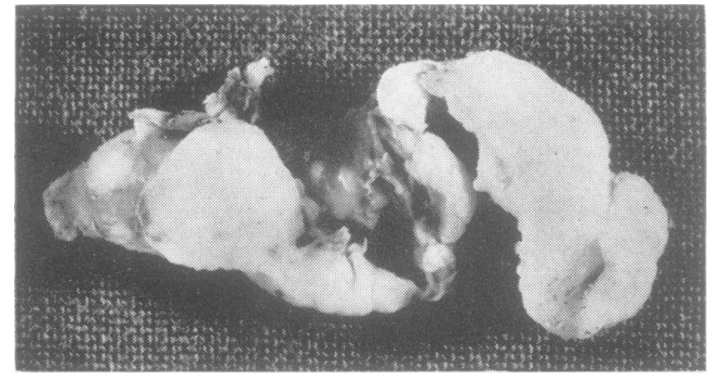

FIG. 3 Thick knobby part of the cyst wall in cross section, showing borders of large and small tuberculomatous caseous areas

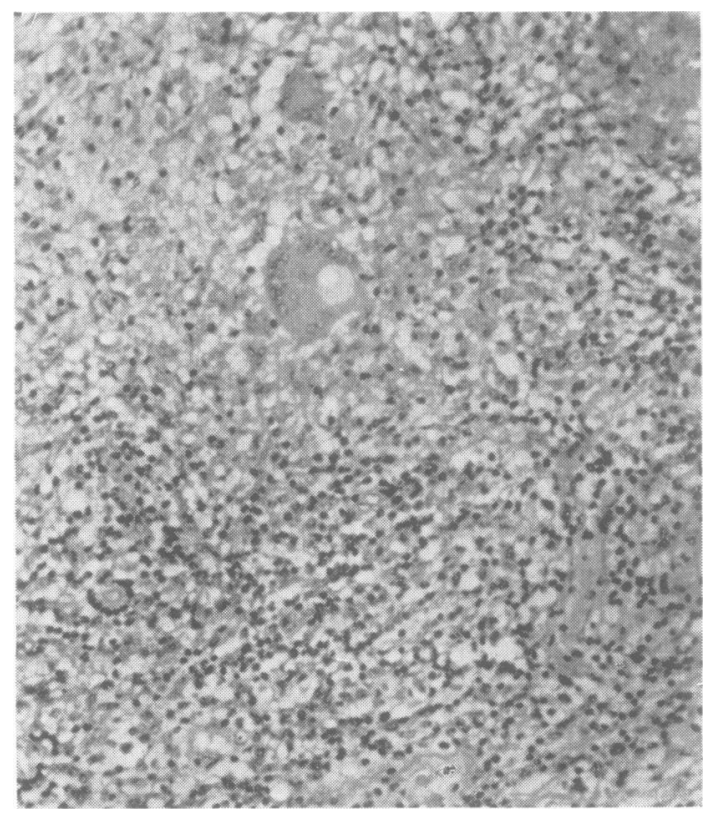

FIG. 4. Haematoxylin-and-eosin-stained section through the border of a tuberculomatous nodule in the cyst wall. Zones of fibrous reaction, of lymphocytic and plasma cell proliferation, and of epithelioid cells and giant cells abutting on to the caseous area can be seen $(\times 175)$.

culosis; he has since been admitted to a tuberculosis hospital.

\section{PATHOLOGICAL FEATURES}

Gross examination of the portions of material received (NP-B-455) showed two types of tissue. There were relatively large solid pieces showing prominent zones of frank caseation bounded by greyish rims of brain tissue, presenting the appear- ance of a tuberculoma. More arresting, however, were portions of cyst wall with small smooth knobby elevations throughout the wall. The inner surface was paler in colour than the outer which bore flecks of clotted blood and stains of altered blood. On making fresh cuts across the wall, two types of tissue could again be recognized, the inner surface being formed of firm white caseous tissue with characteristic crenated borders and the outer of greyish brain tissue. The knobby areas presented in cross sections appearances strongly suggestive of small tuberculomata (Fig. 3).

Microscopic examination of smears prepared from the cyst fluid stained with haematoxylin and eosin and by Papanicolaou's methods showed a large number of lymphocytes together with a fair number of larger mononuclear cells with a less pyknotic or a frankly vesicular nucleus. These cells appeared to be phagocytes since some of them showed ingested cell debris in their cytoplasm. They resembled an 'epithelioid' type of cell. There were plasma cells also. Fibrinous strands were seen throughout the smears and the various reactive cells were often found enmeshed amongst them in small clusters.

Sections stained with haematoxylin and eosin and with Picro-Mallory of both the solid portions and the cyst wall showed identical histological pictures. While the former showed larger areas of caseation surrounded by a thin border of chronic granulomatous reaction, the cyst wall consisted of small circular and ovoid spots of caseation with larger peripheral and intervening areas of granulomatous tissue. The latter consisted of diffuse and tuberculoid accumulations of giant cells, epithelioid cells (some binucleate), plasma cells, and lymphocytes (Fig. 4). Vasculitis and vascular necrosis were clearly seen.

The inner lining of the cyst wall showed in multiple areas a layer, two to many cells deep, of swollen macrophages with granular cytoplasm and one or occasionally more vesicular nuclei. These appeared to be active epithelioid cells. In places where there was a break in continuity of the lining, gitter cells could be observed. The outer zone of the cyst wall was characterized by a proliferation of fibrocytic and astrocytic nuclei and a fibrous stroma. Both these border zones were diffuse and the granulomatous reaction abutted on to them.

Sections stained by the Fite-Faraco method for acid-fast bacilli in tissue sections did not show any mycobacteria.

The gross and microscopic pathological features observed justified the diagnosis of an extensive, chronic, caseous, solid, and cystic, granulomatous process of the nature of a tuberculoma.

The one unusual pathological feature of this 
tuberculoma was the formation of a large cyst within its substance, large enough to accommodate $100 \mathrm{ml}$. of fluid. The cyst fluid was found to contain $200 \mathrm{mg}$. \% of protein and inflammatory and phagocytic cells (described above) which had obviously been shed into the cavity from the granulomatous cyst wall. It is difficult to state definitely what factors combined to produce the cystic change. As there was a history of head injury with unconsciousness, convulsions, and hemiparesis at the start of the illness, trauma was considered as a possible precipitating factor by producing a haemorrhage in or around a tuberculoma, but there was no histological evidence to support this hypothesis. Again, neither the cyst wall nor its contents suggested any resemblance to an acute or chronic tuberculous abscess (Courville, 1945). It was also unlikely that the cystic change was in any way a primary manifestation of infection by Mycobacterium tuberculosis, but rather a secondary necrosis and liquefaction of the central portion of a very large tuberculoma as indicated by the histiocytic and necrotic inner lining of the cyst wall.

The liquefying necrosis and cyst formation, such as that envisaged here, would thus represent an unusual type of secondary change in a tuberculoma in marked contradistinction to the other more common secondary alteration of a regressive nature, which is a fibrosing and calcifying sclerosis of a tuberculoma as discussed by Evans and Courville (1938).

\section{SUMMARY}

The case of a cerebral tuberculoma which had undergone cystic degeneration in a child aged 4 years is presented with a description of the surgical management and the pathological findings. This experience has made us aware that, in the East at least, even a cystic tumour might still be a tuberculoma.

We are grateful to the Dean, K.E.M. Hospital, for permission to publish and to Mr. E. G. Warrier for secretarial assistance.

\section{REFERENCES}

Arseni, C. (1958). J. Neurol. Neurosurg. Psychiat., 21, 308.

Courville, C. B. (1945). Pathology of the Central Nervous System. 2nd Ed. Pacific Press Publishing Association, Mountain View, California.

Cushing, H. (1932). Intracranial Tumours. Thomas, Springfield, Illinois.

Dastur, H. M. (1961). Neurology (Bombay), 9, 83.

Dastur, D. K., and Iyer, C. G. S. (1961). Paper presented at the 1st All India Cancer Conference, Bombay, India.

Dublin, W. B. (1954). Fundamentals of Neuropathology. Thomas, Springfield, Illinois.

Evans, H. S., and Courville, C. B. (1938). Arch. Surg., 36, 637.

Greenfield, J. G., Blackwood, W., McMenemey, W. H., Meyer, A., and Norman, R. M. (1958). Neuropathology. Arnold, London.

Ramamurthi, B., and Varadarajan, M. G. (1961). J. Neurosurg., 18, 1.

Wilson, S. A. Kinnier (1954). Neurology, 2nd Ed., ed. A. Ninian Bruce, Vol. I. Butterworth, London. 